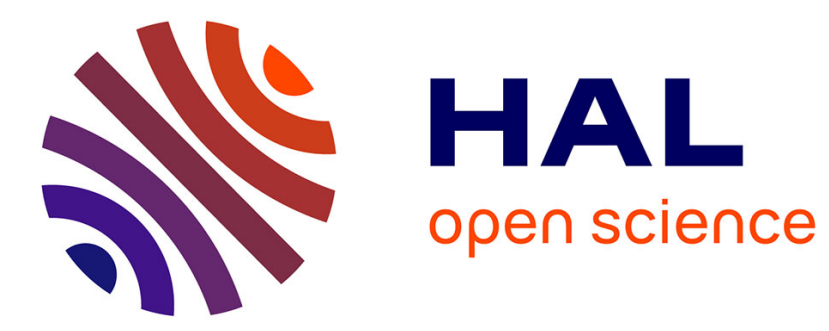

\title{
Meson photoproduction at GRAAL
}

\author{
V. Kouznetsov, O. Bartalini, V. Bellini, J.P. Bocquet, M. Castoldi, A. \\ d'Angelo, J.P. Didelez, R. Di Salvo, A. Fantini, G. Gervino, et al.
}

\section{To cite this version:}

V. Kouznetsov, O. Bartalini, V. Bellini, J.P. Bocquet, M. Castoldi, et al.. Meson photoproduction at GRAAL. International Symposium on Meson-Nucleon Physics and the Structure of the Nucleon 9 MENU 2001, Jul 2001, Washington, United States. pp.160-165. in2p3-00011983

\section{HAL Id: in2p3-00011983 https://hal.in2p3.fr/in2p3-00011983}

Submitted on 24 Oct 2002

HAL is a multi-disciplinary open access archive for the deposit and dissemination of scientific research documents, whether they are published or not. The documents may come from teaching and research institutions in France or abroad, or from public or private research centers.
L'archive ouverte pluridisciplinaire HAL, est destinée au dépôt et à la diffusion de documents scientifiques de niveau recherche, publiés ou non, émanant des établissements d'enseignement et de recherche français ou étrangers, des laboratoires publics ou privés. 


\section{Meson photoproduction at GRAAL}

V. Kouznetsov ${ }^{a, b, *}$, representing the GRAAL Collaboration:

O. Bartalini ${ }^{a, c}$, V. Bellini ${ }^{d}$, J.-P.Bocquet ${ }^{e}$, M. Castoldi ${ }^{f}$, A. D'Angelo ${ }^{a}$, J.-P. Didelez ${ }^{h}$, R. Di Salvo ${ }^{a}$, A. Fantini ${ }^{a}$, G. Gervino ${ }^{g}$, F. Ghio ${ }^{i}$, B. Girolami ${ }^{i}$, M. Guidal ${ }^{h}$, E. Hourany ${ }^{h}$, R. Kunne ${ }^{h}$, A. Lapik ${ }^{b}$, P. Levi Sandri ${ }^{j}$, A. Lleres ${ }^{e}$, D. Moricciani ${ }^{a}$, V. Nedorezov ${ }^{b}$, L. Nicoletti ${ }^{e}$, D. Rebreyend ${ }^{e}$, F. Renard ${ }^{e}$, N. Roudnev ${ }^{k}$, C. Schaerf ${ }^{a}$, M.L. Sperduto ${ }^{d}$, M.C. Sutera ${ }^{d}$, A. Turinge ${ }^{l}$, A. Zabrodin ${ }^{b}$, A. Zucchiatti ${ }^{f}$

${ }^{a}$ University "Tor Vergata" and INFN Sezione di RomaII, I-00133 Rome, Italy

${ }^{b}$ Institute for Nuclear Research, 117312 Moscow, Russia

${ }^{c}$ University of Trento, I-38100 Trento, Italy

${ }^{d}$ INFN, Laboratori Nazionali del Sud, I-95123 Catania, Italy

${ }^{e}$ Institut des Sciences Nucléaires, 38026 Grenoble, Frnace

${ }^{f}$ INFN, Sezione di Genova, I-16146 Genoa, Italy

${ }^{h}$ Institut de Physique Nucléaire, 91406 Orsay, France

${ }^{g}$ INFN, Sezione di Torino and University of Turin, I-10125 Turin, Italy

${ }^{i}$ Instituto Superiore di Sanita and INFN Sezione di RomaI, I-00161 Rome, Italy

${ }^{j}$ INFN, Laboratori Nazionali di Frascati, I-00044 Frascati, Italy

${ }^{k}$ Institute of Theoretical and Experimental Physics, 117259 Moscow, Russia

${ }^{l}$ Kurchatov Institute of Atomic Energy, 123182 Moscow, Russia

*Email: slava@roma2.infn.it, slava@cpc.inr.ac.ru

(Received: 2 November 2001)

The highly polarized and tagged photon beam and the almost $4 \pi$ detector of the GRAAL Collaboration make it possible to produce high quality photoproduction data. Recent results on beam polarization asymmetries $\Sigma$ for $\pi^{+}$and $\eta$ photoproduction, measured over wide angular and energy ranges, are presented. Data of high precision provide important constraints for partial wave analysis.

\section{Introduction}

Probing the nucleon with polarized photons provides important information regarding the spectrum of nucleon excited states. Over past years, the photoproduction of mesons has demonstrated its potential [1-5] as a tool to explore $N^{*}$, complementary to the $\pi N$ scattering. Precise data from modern photon factories essentially impact theoretical studies of nucleon resonances.

Properties of resonances are extracted from the photoproduction data by means of the partial wave analysis and the multipole decomposition in the framework of different approaches [1-5]. The comparison of calculated observables to experimental data constraints theoretical models and determines the role and properties of the included resonances. The extraction of resonances parameters requires both unpolarized cross section data and polarization observables [6]. While the cross section is a source of information on dominating components of the scattering amplitude, the polarization observables are much more sensitive to the non-dominant contributions. For pseudoscalar meson photoproduction, this is well illustrated in terms of four helicity amplitudes $H_{i}$ corresponding to four possible helicity states of the target and recoil nucleon,

$$
\begin{aligned}
\frac{d \sigma}{d \Omega} & \sim\left|H_{1}\right|^{2}+\left|H_{2}\right|^{2}+\left|H_{3}\right|^{2}+\left|H_{4}\right|^{2}, \\
\Sigma & \sim \operatorname{Re}\left(H_{1} H_{4}^{*}-H_{2} H_{3}^{*}\right), \\
T & \sim \operatorname{Im}\left(H_{1} H_{2}^{*}+H_{3} H_{4}^{*}\right), \\
R & \sim \operatorname{Im}\left(H_{1} H_{3}^{*}+H_{2} H_{4}^{*}\right),
\end{aligned}
$$


where the three single-polarization observables $\Sigma, T, R$ are the beam, target and recoil asymmetries, i.e. the measures of anisotropy of a reaction yield in respect to the polarization of the incoming photon, target and recoil nucleons.

The novel GRAAL facility [7] was designed to measure the polarization observables, in particular, the $\Sigma$ beam asymmetry, in photon-induced reactions. During last years, experimental data have been collected for various channels of the photon-nucleon interaction. The recent progress in the analyses of $\pi^{+}$and $\eta$ photoproduction is presented in this report.

\section{The GRAAL setup}

A polarized and tagged photon beam (Fig. 1) at GRAAL is produced by backscattering of laser light on $6.04 \mathrm{GeV}$ electrons which circulate in the storage ring of the ESRF (Grenoble, France). Through the use of green $514 \mathrm{~nm}$ laser light, the tagged spectrum covers an energy range of $0.55-1.1 \mathrm{GeV}$. Alternately, the UV line can be employed, resulting in an energy range of $0.8-1.5 \mathrm{GeV}$. The linear beam polarization varies from $\sim 0.45$ at the lower energy limits to 0.98 at the upper limits. The tagger provides an energy resolution of $16 \mathrm{MeV}$ (FWHM) which is limited by the emittance and energy spread of the electron beam. The $\gamma$ tagging rate is $2 \times 10^{6}$ photons per second for the integrated spectrum. The detection system (Fig. 1) includes three main parts:

- At forward angles $\theta_{l a b} \leq 25^{\circ}$ there are two planar wire chambers, a thin time-of-flight (TOF) hodoscope made up of 26 horizontal and 26 vertical plastic scintillator strips, each $3 \mathrm{~cm}$ thick, and a TOF shower wall [8]. The latter is an assembly of 16 modules, each being a sandwich of four converter-plus-scintillator layers.

- At central angles from $25^{\circ}$ to $155^{\circ}$, the target is surrounded by two cylindrical wire chambers, a $5 \mathrm{~mm}$ thick scintillator $\Delta E$ barrel, and a BGO ball made up of 480 crystals, each of 21 radiation lengths [9].

- At backward angles $\theta_{l a b} \geq 155^{\circ}$ there are two plastic scintillator disks separated by a $1 \mathrm{~cm}$ lead converter.

The apparatus provides the detection and identification of all types of final state particles in an almost $4 \pi$ solid angle.

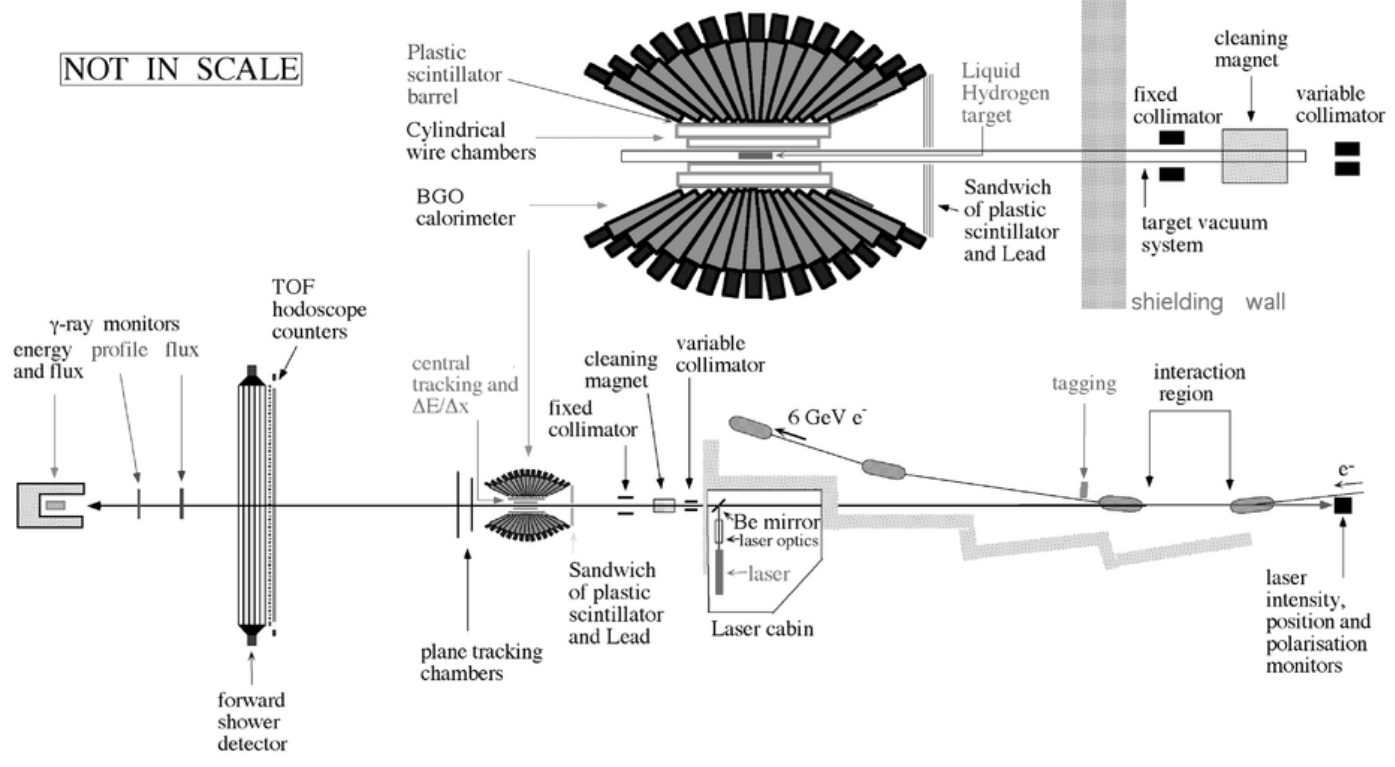

Figure 1: The GRAAL setup. 
The azimuthal symmetry of the GRAAL detector makes it suitable for measurements of the $\Sigma$ beam asymmetry. For photons linearly polarized in the vertical plane with a polarization degree $\mathrm{P}$, the cross section of the reaction under study can be written as

$$
\left(\frac{d \sigma}{d \Omega}\right)_{p o l}(\phi)=\left(\frac{d \sigma}{d \Omega}\right)_{\text {unpol }}[1+P \Sigma \cos (2 \phi)],
$$

where $\phi$ is the angle between the reaction plane and the beam polarization, $\Sigma$ is the beam asymmetry. By switching the beam polarization alternatively between horizontal and vertical states, two independent sets of data can be collected. The sum of two yields normalized by the respective fluxes provides the unpolarized cross section and the possibility to correct the small azimuthal anisotropies in the detector response. The $\Sigma$ beam asymmetry can be extracted from the fit to the $\phi$ distributions of selected events as

$$
\frac{\left(\frac{d \sigma}{d \Omega}\right)_{\text {pol }}(\phi)}{\left(\frac{d \sigma}{d \Omega}\right)_{\text {unpol }}}=1+P \Sigma \cos (2 \phi)=\frac{2 F_{\text {ver }}(\phi)}{F_{\text {ver }}(\phi)+\alpha F_{\text {hor }}(\phi)}
$$

$F_{\text {ver }}$ and $F_{h o r}$ are measured azimuthal distributions of selected events for the vertical and horizontal beam polarizations, $\alpha$ is the ratio of the beam fluxes for each polarization state.

\section{$3 \quad \pi^{+} N$ photoproduction}

Initially, 92 beam asymmetry data for positive pion photoproduction have been measured from 0.6 to $1.05 \mathrm{GeV}$ using the green laser [10]. These data, shown in Fig. 2 together with the most accurate previous results [11-13], are in good agreement with the other experiments. Data points cover an almost unmeasured region of backward angles.

Recently, new data have been obtained at higher energies of $0.8-1.5 \mathrm{GeV}$ using the UV laser [14]. The new data set (Fig. 3) includes 237 beam asymmetries, measured over an angular range of 40$160^{\circ} .136$ data points were produced in an almost unexplored domain above $1.05 \mathrm{GeV}$, where only

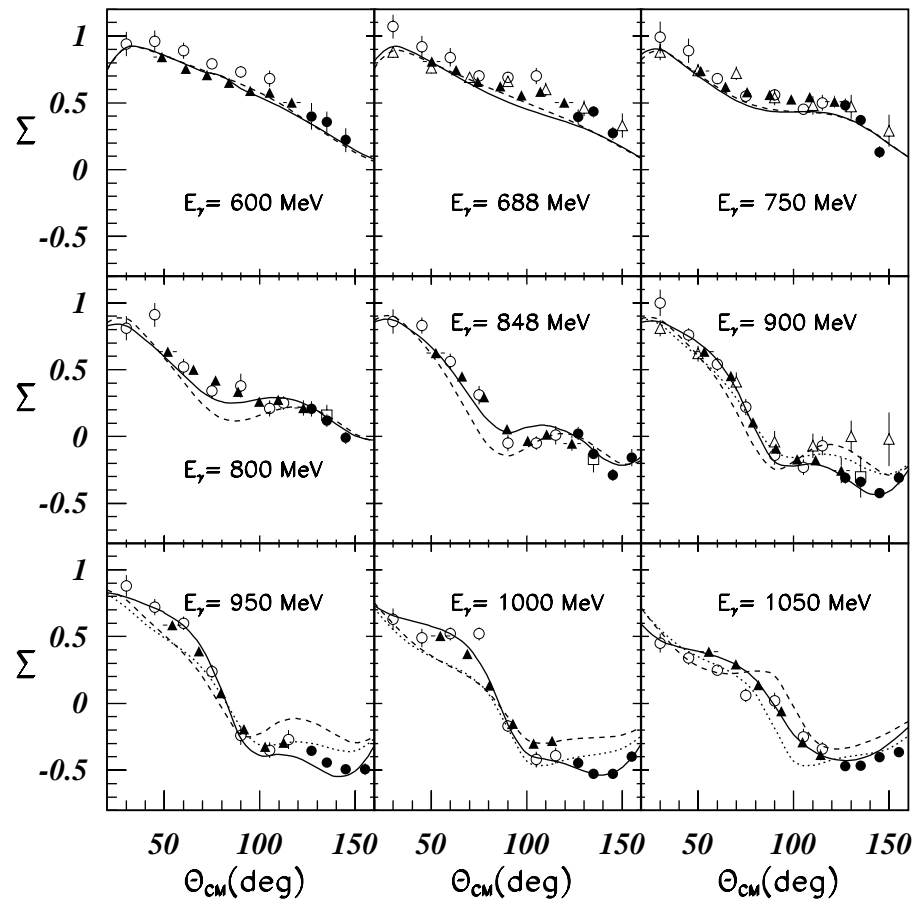

Figure 2: $\Sigma$ beam asymmetry for $\pi^{+} n$ photoproduction. Black circles and triangles indicate the GRAAL results [10], measured with the green laser; open circles indicate the results of the Daresbury group [11]; open triangles and squares indicate the results from SLAC $[12,13]$. Solid lines are the FA01 solution of the SAID partial wave analysis; dashed lines are the predictions of MAID2000 [3]; dotted lines are the results from [15] after fitting the benchmark data base [16]. 


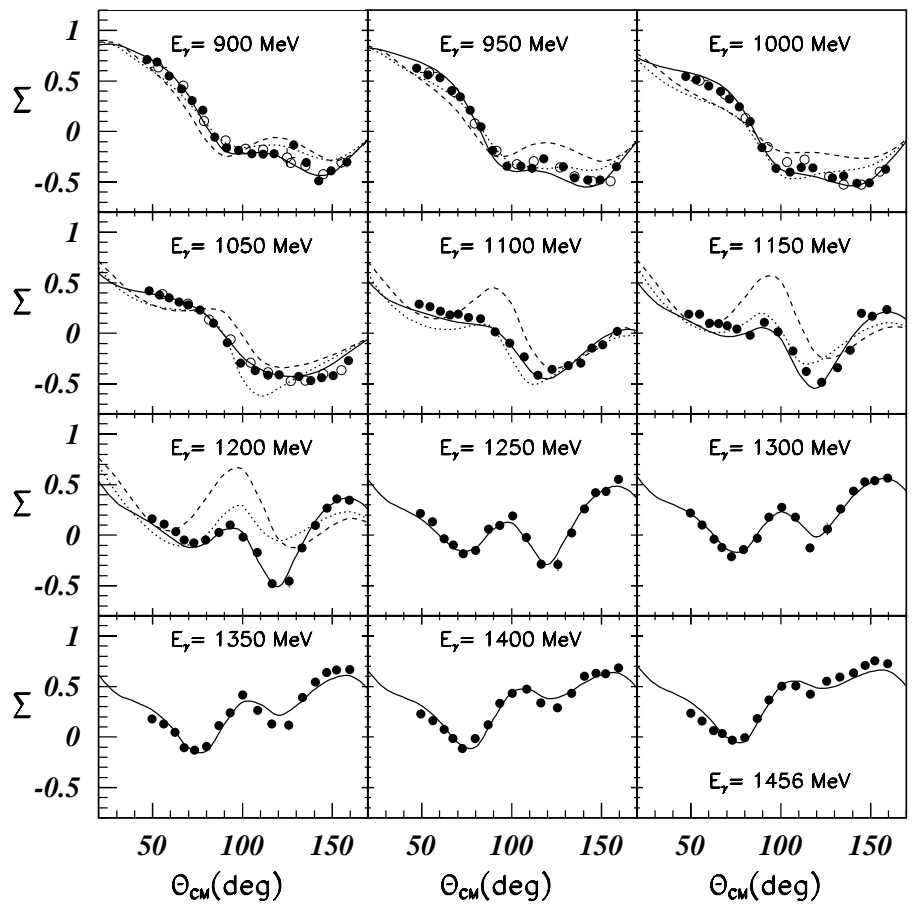

Figure 3: $\Sigma$ beam asymmetry for $\pi^{+} n$ photoproduction. Black circles indicate the new GRAAL results (preliminary), measured with the UV laser; open circles indicate the previous GRAAL results from [10]. Solid lines are the FA01 solution of the SAID partial wave analysis; dashed lines are the predictions of MAID2000 [3]; dotted lines are the results from [15] after fitting the benchmark data base [16].

45 old data of lower accuracy were available. New results also cover backward angles above $120^{\circ}$ where no previous measurements exist. Through the use of the wire chambers in order to determine the pion trajectories, the improved resolution in the determination of the scattering angle $\Theta_{c m}$ of about $3^{\circ}$ have been achieved. This feature has made it possible to produce the data points with narrow angular bins of $6-10^{\circ}$, in order to reveal a complicate angular variation of $\Sigma$.

In Fig. 3, both sets of data are compared at overlapping energies. The data have been obtained using either green or UV lasers, which produce different beam spectra and different polarizations for each beam energy [7]. Given these differences, the reproducibility of our results is excellent and supports the quality of both data sets.

We have compared our results with the predictions of a unitary isobar model MAID2000 [3]. At the energies below $0.95 \mathrm{GeV}$, this model reasonably reproduces our data. At higher energies the difference becomes more pronounced. The latest version of these calculations [15], which includes resonance parameters derived from a fit to the restricted data base [16], exhibits an improvement. Nevertheless, the discrepancy still remains above $1 \mathrm{GeV}$.

The new FA01 solution of the partial wave analysis of the Data Analysis Center of The George Washington University (SAID) have been developed after adding our data to the data base [2]. This solution reproduces our data reasonably well, with $\chi^{2} /$ data of 555/237.

\section{$4 \quad \eta$ photoproduction}

Fig. 4 shows beam asymmetries for $\eta$ photoproduction on the proton, measured at GRAAL three years ago [17]. No other results were available. The measurement have been performed using the green laser from the threshold to $1.1 \mathrm{GeV}$. The results have been produced in two ways: analyzing events, when two or six photons, originating from the $(\eta \rightarrow 2 \gamma)$ and $\left(\eta \rightarrow 3 \pi^{0} \rightarrow 6 \gamma\right)$ decay channels are detected in the BGO ball; and with the detection of one photon from the $(\eta \rightarrow 2 \gamma)$ decay in the forward shower wall and the other in the BGO. Both analyses have provided statistically independent sets of data, which have confirmed each the other. The results have shown the large forward values of the asymmetry $\Sigma$ near $1.05 \mathrm{GeV}$, not predicted by old models. 


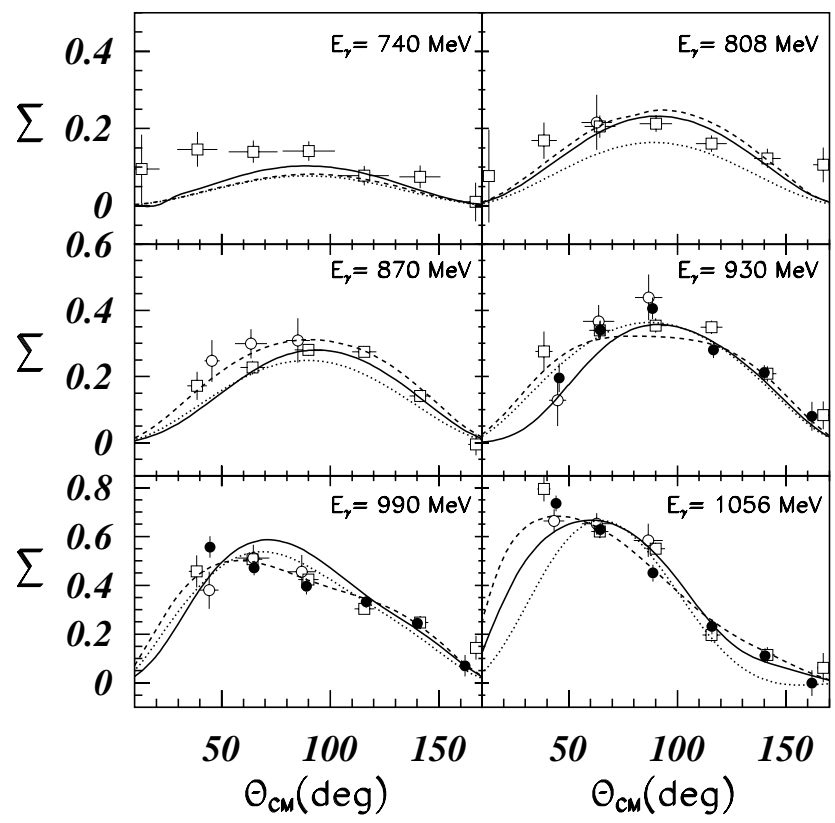

Figure 4: $\Sigma$ beam asymmetry, corresponding to the $\eta$ photoproduction on the proton. Open squares and circles indicate the results from [17] measured using the green laser: the squares correspond to the detection of 2 or $6 \gamma$ 's in the BGO ball; circles correspond to $1 \gamma$ in the forward shower wall, and $1 \gamma$ in the BGO. Black circles are our preliminary results, measured with the UV laser. Solid curves are predictions of the eta-MAID [5]; dashed curves denote the BO12 solution of the SAID analysis [18]; dotted curves are predictions of the quark model of B. Saghai and Z. Li [4].

The analysis of new data, collected with the UV laser at higher energies, aims to reveal more details in the variation of $\Sigma$ at forward angles. One important advantage is the use of the wire chambers to reconstruct the tracks of recoil protons, in order to achieve the best angular resolution in the determination of the scattering angle $\Theta_{\mathrm{cm}}$. Both type of events (2 or $6 \gamma$ 's in the BGO and $1 \gamma$ in the forward wall and $1 \gamma$ in the BGO) are considered together. The latter essentially increases the overall statistics at forward angles. These features make it possible to reduce the widths in the angular binning as compared with the previous data.

In Fig. 4, preliminary results of the new analysis are shown together with the published data using the similar angular bins. As in the case of $\pi^{+} n$ data, the reproducibilty of our results is quite good. The main set of the new results is shown in Fig. 5 . The data exhibit a peak near $\sim 50^{\circ}$ at

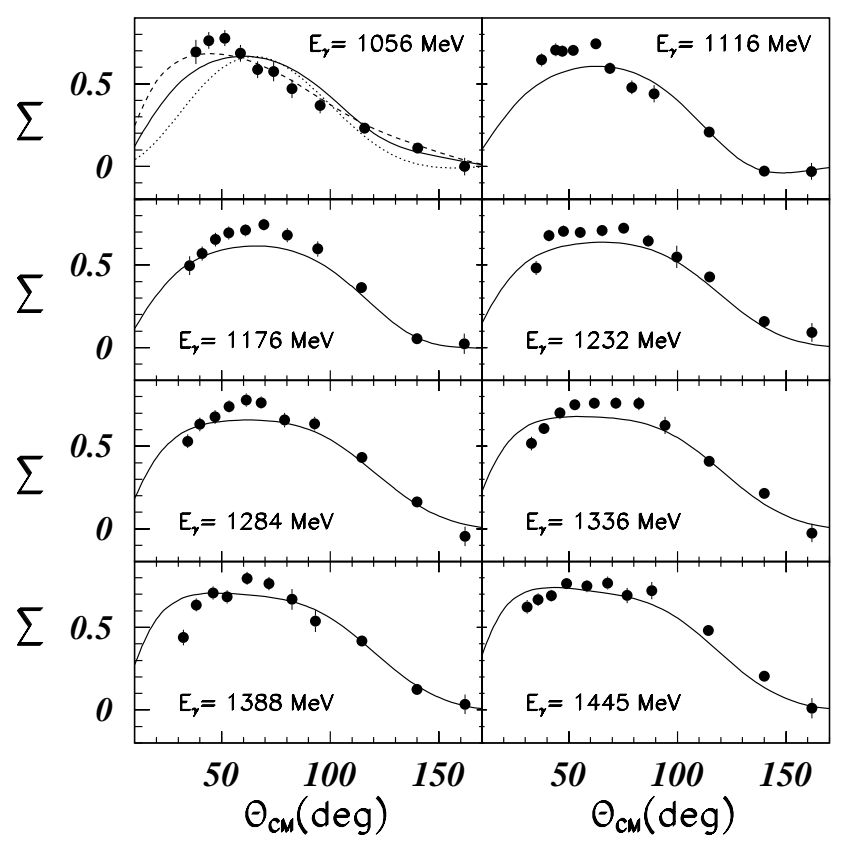

Figure 5: Preliminary results for the $\Sigma$ beam asymmetry observable for the $\eta$ photoproduction on the proton measured using the UV laser. Solid curves are predictions of the eta-MAID [5]; dashed curves denote the BO12 solution of the SAID analysis [18]; dotted curves are predictions of the quark model of B. Saghai and Z. Li [4]. 
the photon energy of $1.05 \mathrm{GeV}$, which was not clear evidenced in the previous results. At higher energies, the peak becomes more spread, showing large values of $\Sigma$ at the angles from 40 to $90^{\circ}$.

Our results are compared with the predictions of several models: the BO12 solution of the SAID partial wave analysis [18]; the quark model of B. Saghai and Z. Li [4]; and the eta-MAID [5]. All the models are rather close to the data below $1.05 \mathrm{GeV}$. However, neither of them well reproduces the observed peculiarity near $50^{\circ}$ at $1.05 \mathrm{GeV}$. At higher energies, the predictions of the eta-MAID model, only available, are in reasonable agreement with our data.

\section{Conclusions}

Over last several years, the GRAAL Collaboration produces polarized photoproduction data of high precision. The recent progress in the study of $\pi^{+}$and $\eta$ photoproduction is reported. New constraints are placed upon partial wave analyses.

Acknowledgments: We are grateful to all theoreticians who were interested in our data. We wish to thank I. Strakovsky, R. Workman, S. Kamalov, B. Saghai, L. Tiator, and W.-T. Chiang for their close co-operation with GRAAL. We acknowledge the machine operation group of the ESRF for providing the stable beam operation, and the staffs of the contributing Institutes for their assistance in the development and maintenance of the apparatus.

\section{References}

[1] R. M. Davidson, N. C. Mukhopadhyay, M. S. Pierce, R. A. Arndt, I. I. Strakovsky, and R. L. Workman, Phys. Rev. C 59, 1059 (1999); L. Tiator, D. Drechsel, G. Knöchlein, and C. Bennhold, Phys. Rev. C 60, 035210 (1999); R. L. Workman, R. A. Arndt, and I. I. Strakovsky, Phys. Rev. C 62, 048201 (2000); T. Mart and C. Bennhold, Phys. Rev. C 61, 012201 (2000); T. Feuster and U. Mosel, Phys. Rev. C 59, 460 (1999).

[2] R. Arndt, I. Strakovsky, and R. Workman, Phys. Rev. C 53, 430 (1996). The SAID solutions and the single-pion photoproduction data base are available via telnet/ssh to gwdac.phys.gwu.edu, user: said.

[3] D. Drechsel, O. Hanstein, S. Kamalov, and L. Tiator, Nucl. Phys. A645, 145 (1999); Predictions are available at http://www.kph.uni-mainz.de/MAID.

[4] B. Saghai and Z. Li, Eur. Phys. J. A 11, 217 (2001).

[5] W.-T. Chiang, S.N. Yang, L. Tiator, and D. Drechsel, E-print Nucl-th/0110034. Predictions are available at http://www.kph.uni-mainz.de/MAID.

[6] W.-T. Chiang and F. Tabakin, Phys. Rev. C 55, 2054 (1997); R. Workman, Few Body Syst. Suppl. 11, 94 (1999).

[7] J. P. Bocquet et al., Nucl. Phys. A622, 125 (1997).

[8] V. Kouznetsov et al., to be published in Nucl. Instr. and Meth.

[9] F. Ghio et al., Nucl. Instr. and Meth. A404, 71 (1998);

[10] J. Ajaka et al., Phys. Lett. B475, 372 (2000).

[11] P. J. Bussey et al., Nucl. Phys. B154, 205 (1979).

[12] G. Knies et al., Phys. Rev. D 10, 2778 (1974).

[13] R. Zdarko and E. Dolly, Nuovo Cimento A10, 10 (1972).

[14] Publication in preparation.

[15] S. Kamalov, private communication; S. Kamalov et al., E-print nucl-th/0106045.

[16] R. A. Arndt et al., "Multipole Analysis of Benchmark Dataset for Pion Photoproduction", to appear in the Proceedings of the Workshop on the Physics of Excited Nucleons (NSTAR 2001), Mainz, Germany, 7-10 March 2001, Eprint nucl-th/0106059.

[17] J. Ajaka et al., Phys. Rev. Lett. 81, 1797 (1998).

[18] R. Arndt, I. Strakovsky, and R. Workman, Bull. Am. Phys. Soc. 45, 34 (2000). 